\begin{tabular}{|c|l|}
\hline Title & Ultrafast ellipsometric interferometry for direct detection of coherent phonon strain pulse profiles \\
\hline Author(s) & $\begin{array}{l}\text { Matsuda, O samu; Tomoda, Motonobu; Tachizaki, Takehiro; Koiwa, Shun; Ono, A tsushi; A oki, Kae; Beardsley, Ryan } \\
\text { P.; Wright, Oliver B. }\end{array}$ \\
\hline Citation & $\begin{array}{l}\text { Journal of the Optical Society of A merica B - Optical Physics, 30(7), 1911-1921 } \\
\text { https://doi.org/10.1364/JOSA B.30.001911 }\end{array}$ \\
\hline Issue Date & 2013-07 \\
\hline Doc URL & http://hdl.handle.net/2115/53078 \\
\hline Rights & ○ 2013 Optical Society of A merica, Inc. \\
\hline Type & article \\
\hline File Information & josab-30-7-1911.pdf \\
\hline
\end{tabular}

Instructions for use 


\title{
Ultrafast ellipsometric interferometry for direct detection of coherent phonon strain pulse profiles
}

\author{
Osamu Matsuda,* Motonobu Tomoda, Takehiro Tachizaki, Shun Koiwa, \\ Atsushi Ono, Kae Aoki, Ryan P. Beardsley, and Oliver B. Wright \\ Division of Applied Physics, Faculty of Engineering, Hokkaido University, Sapporo, Hokkaido 060-8628, Japan \\ *Corresponding author: omatsuda@eng.hokudai.ac.jp
}

Received March 18, 2013; revised April 27, 2013; accepted April 30, 2013; posted May 8, 2013 (Doc. ID 187284); published June 19, 2013

\begin{abstract}
We describe an ultrafast optical technique to quantitatively detect picosecond ultrasonic displacements of solid surfaces, thus giving access to the longitudinal strain pulse shape. Transient optical reflectance changes recorded at oblique optical incidence with a common-path interferometric configuration based on ultrafast ellipsometry monitor gigahertz coherent phonon pulses. We demonstrate for a tungsten film the quantitative extraction of the strain pulse shape free of distortions arising from the photoelastic effect, and analyze the results with the two-temperature model to obtain the value $g \approx 3 \times 10^{17} \mathrm{Wm}^{-3} \mathrm{~K}^{-1}$ for the electron-phonon coupling constant. Analysis of the data also reveals a thermo-optic contribution. (C) 2013 Optical Society of America

OCIS codes: (120.3180) Interferometry; (120.2130) Ellipsometry and polarimetry; (320.0320) Ultrafast

optics; (320.5390) Picosecond phenomena; (120.0120) Instrumentation, measurement, and metrology.

http://dx.doi.org/10.1364/JOSAB.30.001911
\end{abstract}

\section{INTRODUCTION}

Picosecond pump light pulses incident on opaque solid or liquid media can generate coherent phonon pulses in the gigahertz to terahertz range. Their propagation can be monitored with delayed probe light pulses as transient optical reflectance changes. This pulse-echo technique is referred to as picosecond laser ultrasonics, and has been used to study material properties and the vibrations of nanostructures [1-28]. Picosecond laser ultrasonics has also proved useful in elucidating the ultrashort dynamics of excited electrons $[\underline{4}, \underline{5}, 7,12,13,17,22]$. For such quantitative studies, it is crucial to know the precise shape of the propagating acoustic strain pulses.

It is difficult in general, however, to measure the strain pulse shape because of the photoelastic interaction between the probe light and the sample, that is, the coupling of the optical indices to the strain. The probe light penetrates into the solid and monitors the strain over a finite region. This leads to a smoothing effect that normally prevents the exact strain pulse shape from being measured. With noninterferometric optical probing, only in certain limiting cases, for example, when the strain pulse spatial extent is much larger than the optical penetration depth, can the strain pulse shape be accurately measured [22].

Some steps toward the goal of measuring the strain pulse shape in laser picosecond ultrasonics were made when the detection technique was generalized to include optical beam deflection or distortion caused by surface displacements $[\underline{3}, \underline{5}, \underline{29}]$. For longitudinal coherent phonon pulses, the surface displacement temporal variation can be used to extract the strain pulse profile [3]. However, although the surface displacement can be approximately monitored by these deflection or distortion methods, the radial variation of the probe reflectance over the optical pump spot caused by the photoelastic effect contributes to the recorded signals, thus preventing an accurate measurement of strain pulse profiles. A similar problem arises in the use of conventional (normal-incidence) interferometric detection [6,8,9]. In such detection setups, the surface displacement modulates the phase of the reflected probe light. For measurements at normal optical incidence, the phase modulation by the surface displacement is, again, always contaminated by the photoelastic effect.

Following these advances, a theoretical method for the uncontaminated extraction of ultrashort-timescale surface displacements with $s$ - and $p$-polarized probe light at oblique incidence was proposed [30]. This method, however, involves two separate (i.e., $s$ and $p$ optical polarization) measurements and numerical analysis requiring their subtraction, thus compromising the method's potential accuracy. To avoid this problem an interferometric method was later proposed that is functionally equivalent to the use of $s$ - and $p$-polarized light measurements, but only requiring a single measurement with obliquely incident light [31]. In this paper, we describe in detail and experimentally demonstrate for the first time this ellipsometry-based [32-37] interferometric method by monitoring gigahertz longitudinal coherent-phonon generation and propagation in a tungsten film. We interpret the echo shapes obtained by implementing a two-temperature model of the electron relaxation in tungsten.

In Section $\underline{2}$ we outline the required light-scattering theory and the principle of the separation of the surface displacement and photoelastic contributions. Section 3 concerns the common-path interferometer setup, Section $\underline{4}$ the 
experimental results, and Section $\underline{5}$ their analysis. A brief summary is given in Section 6 .

\section{THEORY}

In a typical measurement in picosecond laser ultrasonics, the pump light is focused onto the sample surface to a spot with a radius $\sim 10 \mu \mathrm{m}$, much larger than the depth usually important for acoustic wave propagation (typically $<1 \mu \mathrm{m}$ ). The spatial divergence of the generated acoustic waves can therefore be neglected and, in homogeneous materials, plane wave acoustic propagation along the depth direction can be assumed. In addition, in isotropic materials, the only significant contribution to the generated acoustic waves comes from longitudinal polarization.

The propagating acoustic waves displace the surface position and modulate the permittivity of the sample through the photoelastic effect. Probe light, focused to the same region as the pump, can detect these permittivity changes through amplitude and phase modulations of the reflected probe light, and also detect the surface displacement through phase modulation. This latter modulation can also be effectively regarded as a permittivity modulation [38]. For depths smaller than the probe spot radius, the focused probe light can be considered to be a plane wave in the sample.

We further assume that the probe light is monochromatic with angular frequency $\omega$ or wavenumber $k=\omega / c$. Since the acoustic frequency, up to terahertz-order in general, is much lower than the optical frequency, the modulation can be regarded as quasi-static, that is, the optical frequency of the reflected probe light is unchanged. The modulation of the reflected probe light electric field $\mathbf{E}(\mathbf{r}, t) \exp (-i \omega t)$ is then obtained by solving the quasi-static wave equation

$$
\left\{\nabla^{2}-\operatorname{grad} \operatorname{div}+k^{2} \varepsilon(\mathbf{r}, t)\right\} \mathbf{E}(\mathbf{r}, t)=0,
$$

where $\varepsilon$ is a permittivity tensor including all perturbations caused by the acoustic wave propagation.

Suppose that a semi-infinite opaque isotropic homogeneous medium occupies $z>0$ and a vacuum occupies $z<0$. The permittivity modulation is assumed to be a function of coordinate $z$ because of the one-dimensional nature of the problem we are describing.

Our oblique probe incidence exploits the polarization dependence of the probing process. The $x$ axis is taken along the sample surface and within the plane of optical incidence, so that the $x$ component of the incident probe light wave vector $\mathbf{k}$ is positive $\left(k_{x}>0\right.$ and $\left.k_{y}=0\right)$. Because of the lateral homogeneity of the system in the $x-y$ plane, the electric field of the probe light takes the form

$$
\mathbf{E}(\mathbf{r}, t)=\mathbf{E}(z, t) \exp \left(i k_{x} x\right)
$$

that is, $k_{x}$ is conserved for the incident, reflected, and transmitted probe light. The wave equation, Eq. (1), is then simplified to

$$
\left\{L\left(k_{x}\right)+k^{2} \varepsilon(z, t)\right\} \mathbf{E}(z, t)=0,
$$

where the operator $L\left(k_{x}\right)$ is defined as

$$
L\left(k_{x}\right) \equiv\left(\begin{array}{ccc}
\frac{\partial^{2}}{\partial z^{2}} & 0 & -i k_{x} \frac{\partial}{\partial z} \\
0 & \frac{\partial^{2}}{\partial z^{2}}-k_{x}^{2} & 0 \\
-i k_{x} \frac{\partial}{\partial z} & 0 & -k_{x}^{2}
\end{array}\right)
$$

The permittivity $\varepsilon(z, t)$ can be expressed as the sum of a homogeneous part $\varepsilon_{h}(z)$ and an inhomogeneous perturbation $\Delta \varepsilon(z, t)$ :

$$
\varepsilon(z, t)=\varepsilon_{h}(z)+\Delta \varepsilon(z, t) .
$$

$\varepsilon_{h}(z)$ is equal to the permittivity of the vacuum, i.e., unity, for $z<0$, and to the permittivity of the medium, $\varepsilon_{1}$, for $z>0$. $\Delta \varepsilon(z, t)$ includes all other spatiotemporally varying perturbations, including the photoelastic effect and the surface displacement.

In the case $\Delta \varepsilon(z, t)=0$ (i.e., the nonperturbed system), Equations (3)-(5) give the total electric field of the incident, reflected, and transmitted probe light for $s$-polarized incident light of unit amplitude

$$
\mathbf{E}_{0 s}=\left\{\begin{array}{cc}
\left(\exp \left(i k^{\prime} z\right)+r_{s} \exp \left(-i k^{\prime} z\right)\right) \mathbf{e}_{s} & (z<0) \\
t_{s} \exp \left(i k^{\prime \prime} z\right) \mathbf{e}_{s} & (z>0)
\end{array}\right.
$$

where

$$
\mathbf{e}_{s}=\left(\begin{array}{l}
0 \\
1 \\
0
\end{array}\right)
$$

is the normalized polarization vector,

$$
r_{s}=\frac{k^{\prime}-k^{\prime \prime}}{k^{\prime}+k^{\prime \prime}}, \quad t_{s}=\frac{2 k^{\prime}}{k^{\prime}+k^{\prime \prime}}
$$

are the amplitude reflectance and transmittance, respectively, and

$$
k^{\prime}=\left(k^{2}-k_{x}^{2}\right)^{1 / 2}, \quad k^{\prime \prime}=\left(\varepsilon_{1} k^{2}-k_{x}^{2}\right)^{1 / 2}
$$

are the $z$ components of the wave vectors in vacuum and in the medium, respectively. Similarly, the total electric field for $p$-polarized incident light of unit amplitude is given by

$$
\mathbf{E}_{0 p}=\left\{\begin{array}{cc}
\exp \left(i k^{\prime} z\right) \mathbf{e}_{i p}+r_{p} \exp \left(-i k^{\prime} z\right) \mathbf{e}_{r p} & (z<0) \\
t_{p} \exp \left(i k^{\prime \prime} z\right) \mathbf{e}_{t p} & (z>0)
\end{array}\right.
$$

where

$\mathbf{e}_{i p}=\frac{1}{k}\left(\begin{array}{c}k^{\prime} \\ 0 \\ -k_{x}\end{array}\right), \quad \mathbf{e}_{r p}=\frac{1}{k}\left(\begin{array}{c}-k^{\prime} \\ 0 \\ -k_{x}\end{array}\right), \quad \mathbf{e}_{t p}=\frac{1}{\varepsilon_{1}^{1 / 2} k}\left(\begin{array}{c}k^{\prime \prime} \\ 0 \\ -k_{x}\end{array}\right)$

are the normalized polarization vectors for the incident, reflected, and transmitted light, and

$$
r_{p}=\frac{\varepsilon_{1} k^{\prime}-k^{\prime \prime}}{\varepsilon_{1} k^{\prime}+k^{\prime \prime}}, \quad t_{p}=\frac{2 \varepsilon_{1}^{1 / 2} k^{\prime}}{\varepsilon_{1} k^{\prime}+k^{\prime \prime}}
$$

are the amplitude reflectance and transmittance, respectively. 
With a small, finite perturbation $\Delta \varepsilon(z, t) \neq 0$, the total electric field becomes

$$
\begin{aligned}
\mathbf{E}(z, t) & =\mathbf{E}_{0}(z)+\int_{-\infty}^{\infty} k^{2} G\left(z, z^{\prime}\right) \Delta \varepsilon\left(z^{\prime}, t\right) \mathbf{E}\left(z^{\prime}, t\right) \mathrm{d} z^{\prime} \\
& \simeq \mathbf{E}_{0}(z)+\int_{-\infty}^{\infty} k^{2} G\left(z, z^{\prime}\right) \Delta \varepsilon\left(z^{\prime}, t\right) \mathbf{E}_{0}\left(z^{\prime}\right) \mathrm{d} z^{\prime}
\end{aligned}
$$

to first-order. The vector $\mathbf{E}_{0}$, corresponding to the polarization of the incident light, is a linear combination of Eqs. (6) and (8). $G\left(z, z^{\prime}\right)$ is a $3 \times 3$ Green's function matrix which satisfies

$$
\left\{L\left(k_{x}\right)+k^{2} \varepsilon_{h}(z)\right\} G\left(z, z^{\prime}\right)=-\delta\left(z-z^{\prime}\right) I,
$$

where $I$ is the $3 \times 3$ identity matrix. For the system in question, the nonzero components of $G\left(z, z^{\prime}\right)$ are given by

$$
\begin{aligned}
& G_{x x}\left(z, z^{\prime}\right)=\frac{i k^{\prime} k^{\prime \prime}}{k^{2}\left(\varepsilon_{1} k^{\prime}+k^{\prime \prime}\right)} \exp \left\{i\left(k^{\prime \prime} z^{\prime}-k^{\prime} z\right)\right\}, \\
& G_{x z}\left(z, z^{\prime}\right)=\frac{i k^{\prime} k_{x}}{k^{2}\left(\varepsilon_{1} k^{\prime}+k^{\prime \prime}\right)} \exp \left\{i\left(k^{\prime \prime} z^{\prime}-k^{\prime} z\right)\right\}, \\
& G_{y y}\left(z, z^{\prime}\right)=\frac{i}{k^{\prime}+k^{\prime \prime}} \exp \left\{i\left(k^{\prime \prime} z^{\prime}-k^{\prime} z\right)\right\}, \\
& G_{z x}\left(z, z^{\prime}\right)=\frac{i k^{\prime \prime} k_{x}}{k^{2}\left(\varepsilon_{1} k^{\prime}+k^{\prime \prime}\right)} \exp \left\{i\left(k^{\prime \prime} z^{\prime}-k^{\prime} z\right)\right\}, \\
& G_{z z}\left(z, z^{\prime}\right)=\frac{i k_{x}^{2}}{k^{2}\left(\varepsilon_{1} k^{\prime}+k^{\prime \prime}\right)} \exp \left\{i\left(k^{\prime \prime} z^{\prime}-k^{\prime} z\right)\right\},
\end{aligned}
$$

for the region of $z<0$ and $z^{\prime}>0[\underline{21}, \underline{30}, \underline{39}] . \Delta \varepsilon(z, t)$ is, for the time being, assumed to be composed of a sum of the photoelastic contribution $\varepsilon_{\mathrm{pe}}$ and the surface displacement contribution $\varepsilon_{\mathrm{d}}$. Other contributions will be considered later. For an isotropic medium and $z$-propagating longitudinal acoustic waves, the photoelastic contribution is given by

$$
\varepsilon_{\mathrm{pe}}(z, t)=\left(\begin{array}{ccc}
P_{12} & 0 & 0 \\
0 & P_{12} & 0 \\
0 & 0 & P_{11}
\end{array}\right) \eta_{z z}(z, t)
$$

where $\eta_{z z}$ is the $z z$ strain component, and $P_{11}$ and $P_{12}$ are photoelastic tensor components in simplified notation, corresponding to $P_{1111}$ and $P_{1122}$, respectively, in full tensor notation. The surface displacement contribution is given by

$$
\varepsilon_{\mathrm{d}}(z, t)=\left\{\begin{array}{cc}
\left(\varepsilon_{1}-1\right) I & \left(u_{0}(t)<z<0\right) \\
\left(1-\varepsilon_{1}\right) I & \left(0<z<u_{0}(t)\right) \\
0 & \text { otherwise }
\end{array}\right.
$$

where $u_{0}(t)$ is the $z$ component of the displacement at the surface $(z=0)$.

When $\left|u_{0}(t)\right|$ is much smaller than the optical wavelength, Eq. (9) is simplified to

$$
\begin{aligned}
\mathbf{E}(z, t) \simeq & \mathbf{E}_{0}(z)+\int_{0}^{\infty} k^{2} G\left(z, z^{\prime}\right) \varepsilon_{\mathrm{pe}}\left(z^{\prime}, t\right) \mathbf{E}_{0}\left(z^{\prime}\right) \mathrm{d} z^{\prime} \\
& +k^{2} u_{0}(t) G(z,+0)\left(1-\varepsilon_{1}\right) \mathbf{E}_{0}(-0)
\end{aligned}
$$

Because of the discontinuity of $\mathbf{E}_{0}$ and $G$ at $z=0$, these values for the left $(-0)$ and right $(+0)$ limits toward $z=0$ should be properly distinguished. Substituting Eqs. (6), (8), (11), and
(12) into Eq. (14), the relative reflectance changes, $\delta r_{s} / r_{s}$ and $\delta r_{p} / r_{p}$, can be obtained. The resulting total electric field in the region $z<0$ for $s$-polarized incident light is given by

$$
\mathbf{E}=\left(\exp \left(i k^{\prime} \boldsymbol{z}\right)+\left(r_{s}+\delta r_{s}\right) \exp \left(-i k^{\prime} \boldsymbol{z}\right)\right) \mathbf{e}_{s},
$$

where

$$
\frac{\delta r_{s}}{r_{s}}=2 i k^{\prime} u_{0}(t)+\frac{2 i k^{\prime} P_{12}}{1-\varepsilon_{1}} \int_{0}^{\infty} \eta_{z z}\left(z^{\prime}, t\right) \exp \left(2 i k^{\prime \prime} z^{\prime}\right) \mathrm{d} z^{\prime} .
$$

Similarly, that for $p$-polarized incident light is given by

$$
\mathbf{E}=\exp \left(i k^{\prime} z\right) \mathbf{e}_{i p}+\left(r_{p}+\delta r_{p}\right) \exp \left(-i k^{\prime} z\right) \mathbf{e}_{r p}
$$

where

$$
\begin{aligned}
\frac{\delta r_{p}}{r_{p}}= & 2 i k^{\prime} u_{0}(t)+\frac{2 i k^{\prime}\left(P_{12} k^{\prime 2}-P_{11} k_{x}^{2}\right)}{k^{\prime 2}-\varepsilon_{1}^{2} k^{\prime 2}} \\
& \times \int_{0}^{\infty} \eta_{z z}\left(z^{\prime}, t\right) \exp \left(2 i k^{\prime \prime} z^{\prime}\right) \mathrm{d} z^{\prime}
\end{aligned}
$$

Experimentally, the real and imaginary parts of the reflectance change $\delta r / r$ are independently obtainable by the use of an interferometer $[6,8,9]$. However, measurement with a single polarization configuration, $\delta r_{p} / r_{p}$ or $\delta r_{s} / r_{s}$ for example, is not sufficient to separate the surface displacement contribution from the photoelastic contribution, since the imaginary part of the reflectance change contains both contributions.

This difficulty can be circumvented by the measurement of reflectance changes corresponding to two different polarization configurations together: $\delta r_{p} / r_{p}$ and $\delta r_{s} / r_{s}$. Equations (15) and (16) can be rewritten in condensed form as follows:

$$
\begin{aligned}
& \frac{\delta r_{s}}{r_{s}}=i A+c_{s} B, \\
& \frac{\delta r_{p}}{r_{p}}=i A+c_{p} B,
\end{aligned}
$$

where $A$ is the real quantity $2 k^{\prime} u_{0}(t), B$ is the integral appearing in Eqs. (15) and (16), and $c_{s}, c_{p}$ are the factors in front of these integrals, i.e.,

$$
c_{s}=\frac{2 i k^{\prime} P_{12}}{1-\varepsilon_{1}}, \quad c_{p}=\frac{2 i k^{\prime}\left(P_{12} k^{\prime 2}-P_{11} k_{x}^{2}\right)}{k^{\prime 2}-\varepsilon_{1}^{2} k^{\prime 2}} .
$$

Using Eq. (17), it is possible to cancel the quantity $B$ and retrieve $A$ from the independently observed variations $\delta r_{s} / r_{s}$ and $\delta r_{p} / r_{p}$ [31]. However, as previously mentioned, the subtraction of these quantities obtained from two different measurements to derive the surface displacement compromises accuracy.

Direct measurement of the surface displacement can be achieved by the procedure schematically outlined in Fig. 1 . Consider the use of linearly polarized probe light with electric field amplitudes $E_{s}$ and $E_{p}$ for the $s$ - and $p$-polarized components, respectively. As described later, it is possible to make an optical setup which interferes $s$ - and $p$-polarized probelight reflection components with an arbitrary phase retardation imposed between them. The resulting electric field amplitude is

$$
E_{\mathrm{int}}=E_{s} r_{s}\left(1+i A+c_{s} B\right)+\mu E_{p} r_{p}\left(1+i A+c_{p} B\right),
$$




$$
\begin{aligned}
& E_{p} r_{p}\left(1+i A+c_{p} B\right) \\
& \text { p-polarized probe } \\
& \text { phase shifter } \stackrel{\checkmark}{\times \mu} \\
& \begin{array}{l|l}
\mu E_{p} r_{p} c_{p} \\
+E_{s} r_{s} c_{s}=0
\end{array} \\
& \begin{array}{lll}
E_{s} r_{s}\left(1+i A+c_{s} B\right) & \left(\mu E_{p} r_{p}+E_{s} r_{s}\right)(1+i+i A) \\
\cline { 1 - 2 } \text { s-polarized probe } & I_{2} \propto 2\left|\mu E_{p} r_{p}+E_{s} r_{s}\right|^{2}(1+A)
\end{array} \\
& \text { to photodetector }
\end{aligned}
$$

Fig. 1. Schematic diagram showing the procedure for direct measurement of surface displacement. Expressions refer to the complex amplitude of the light, except $I_{1}$ and $I_{2}$, which refer to the intensity.

where $\mu=\exp (i \phi)$ is an adjustable phase factor. If $E_{s}, E_{p}$, and $\mu$ are chosen to satisfy $\mu E_{p} r_{p} c_{p}=-E_{s} r_{s} c_{s}$, the amplitude becomes

$$
E_{\mathrm{int}}=\left(E_{s} r_{s}+\mu E_{p} r_{p}\right)(1+i A)
$$

in which the photoelastic contribution is canceled out. Since $A$ is a real quantity, the intensity of the reflected probe light is given by

$$
I_{1} \propto\left|\left(E_{s} r_{s}+\mu E_{p} r_{p}\right)\right|^{2},
$$

independent of $A$, where a small second-order term proportional to $A^{2}$ is omitted. It is, however, possible to retrieve an intensity modulation proportional to $A$ by use of a second interference with reference light exhibiting a $\pi / 2$ phase difference with respect to the unperturbed probe light electric field $E_{s} r_{s}+\mu E_{p} r_{p}$, yielding an intensity of detected probe light given by

$$
\begin{aligned}
I_{2} & \propto\left|\left(E_{s} r_{s}+\mu E_{p} r_{p}\right)(1+i+i A)\right|^{2} \\
& =2\left|E_{s} r_{s}+\mu E_{p} r_{p}\right|^{2}(1+A) .
\end{aligned}
$$

A linear term in $A$ now appears in the intensity, allowing the temporal variation of the surface displacement to be followed. We implement this method in the present paper.

To carry out the above procedure, the condition $c_{p} \neq c_{s}$ should hold; otherwise the reflected probe light disappears when the cancellation condition is fulfilled. This is not problematic in general because $c_{p}$ and $c_{s}$ depend on $k_{x}$, i.e., on the probe-light incident angle, in different ways. It is therefore always possible to realize the condition $c_{p} \neq c_{s}$ by choosing this angle appropriately.

\section{EXPERIMENTAL SETUP}

To demonstrate the above method, we shall apply an appropriate interferometric setup to the measurement of a tungsten film sample. Tungsten is chosen because it is acoustically and optically isotropic in the presence of texture, even in the case of elongated or aligned crystalline grains [40]. It is also advantageous for comparison purposes that several previous reports involving picosecond laser ultrasonics on tungsten films are available $[\underline{7,}, \underline{8}, \underline{11}, \underline{17}, \underline{29}, \underline{41}]$. The film is deposited on a crown-glass substrate (of $1 \mathrm{~mm}$ thickness) by radio frequency sputtering. The nominal film thickness is $300 \mathrm{~nm}$, and the root-mean-square surface roughness is measured by atomic-force microscopy to be $\sim 9 \mathrm{~nm}$ [42]. The ultrasonic measurements involve the use of a mode-locked Ti-sapphire laser with a repetition frequency of $80 \mathrm{MHz}$, a pulse duration of $200 \mathrm{fs}$, and a central wavelength of $830 \mathrm{~nm}$. The fundamental beam (of wavelength of $830 \mathrm{~nm}$ ) is focused onto the sample surface (the film side) with a spot diameter of $20 \mu \mathrm{m}$ at full width at half-maximum (FWHM). This pump light, of fluence $25 \mu \mathrm{J} \mathrm{cm}^{-2}$ per pulse, thermoelastically generates the longitudinal coherent phonon pulses in the sample. Frequencydoubled probe light (of wavelength of $415 \mathrm{~nm}$ ) generated by a $\beta$ - $\mathrm{BaB}_{2} \mathrm{O}_{4}$ crystal, of fluence $50 \mu \mathrm{J} \mathrm{cm}^{-2}$ per pulse, is focused onto the sample surface with a FWHM spot diameter of $8 \mu \mathrm{m}$. The probe light detects transient optical reflectance changes using the interferometer setup described in this paper. The weaker pump light fluence compared to that of the probe does not pose any specific problems, because the interferometer output was found to be linear in both pump and probe fluences. The arrival of the probe light pulses at the sample is varied over the range $0-400$ ps using an optical delay line.

Figure 2 shows the details of the interferometer. The probe light, after passing through the delay line, traverses a polarizer (POL1) and then a half-wave plate (HWP1) to make it linearly polarized at $45^{\circ}$ to the plane of the optical bench (the horizontal plane). A polarizing beam splitter (PBS) then divides the light into horizontally and vertically polarized components, which propagate counterclockwise and clockwise, respectively, in the loop defined by the PBS, the sample, and the two mirrors. The former light pulses (the reference pulses) reach the sample about 1 ns before the latter (the probe pulses). The pump light pulses reach the sample between the arrival of the reference and probe pulses. The ratio between $E_{s}$ and $E_{p}$ for the probe light is adjusted by a half-wave

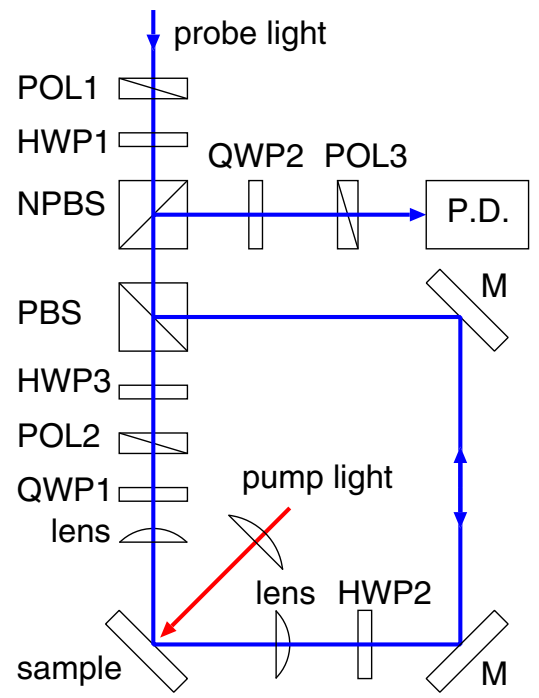

Fig. 2. Schematic diagram of the interferometer setup. HWP, halfwave plate; QWP, quarter-wave plate; PBS, polarizing beam splitter; NPBS, nonpolarizing beam splitter; POL, polarizer; P.D., photodetector; and M, mirror. 
plate (HWP2). The probe light reflected at the sample passes through a quarter-wave plate (QWP1), a polarizer (POL2), and a half-wave plate (HWP3). The optical axes of QWP1 are set at $45^{\circ}$ to the horizontal plane so that the $s$ - and $p$-polarized reflected probe light components interfere with one another with an arbitrary phase retardation determined by the rotation angle of POL2 (see Appendix A). HWP3 is set to rotate the polarization plane of the probe light exiting POL2 to be horizontal, so that the probe light traverses the PBS efficiently. The counterclockwise propagating reference light passes HWP3, POL2, QWP1, sample, and HWP2, and then its vertically polarized component is reflected at the PBS. The clockwise-propagating (horizontally polarized) probe light pulses and counterclockwise-propagating (vertically polarized) reference light pulses are unified at the PBS, are reflected off a nonpolarizing beam splitter (NPBS), are interfered at a quarter-wave plate (QWP2) and a polarizer (POL3), and are finally fed to the photodetector. Because of the common-path configuration, the counterpropagating probe pulses are automatically temporally overlapped at the photodetector, and the optical system is insensitive to mechanical vibrations. The optical axes of QWP2 are set at $45^{\circ}$ to the horizontal plane so that an arbitrary phase retardation between the probe and reference light is introduced by rotating POL3. A phase retardation of $\pm \pi / 2$, achieved by setting POL3 to pass the vertical or horizontal polarization component, provides the optimum interference condition.

The intensity variation $\Delta I$ of the probe light caused by the $\sim$ pm surface displacement is typically very small, $\Delta I / I \sim 10^{-6}$. To observe this variation with sufficient signal-to-noise ratio, the pump light pulses are modulated at $1 \mathrm{MHz}$ with an acousto-optic modulator, and the modulation in the photodetector output at this frequency is detected by a lock-in amplifier. The required bandwidth for the photodetector is thus in the $\mathrm{MHz}$ range (about $2 \mathrm{MHz}$ in the present case).

The setup can be also easily converted for obliqueincidence interferometric measurements $[6,9,43]$. For this purpose, QWP1 and POL2 are removed. The polarization of the probe light is selected to be either $s$ or $p$ by the use of HWP2 and HWP3. According to the position of POL3, the reflected probe light intensity is chosen in turn to be proportional to either $\rho_{j}+\delta \phi_{j}$ or $\rho_{j}-\delta \phi_{j}$, where $\rho_{j}$ and $\delta \phi_{j}$ are the real and imaginary parts of the relative reflectance change $\delta r_{j} / r_{j}$ for the polarization $j=p, s$.

For a simple reflectivity-change measurement of only $\rho_{j}$, QWP2 is further removed and POL3 is set to pass horizontally polarized light. This ensures that only probe light in the clockwise path is used. The polarization of the probe light can be chosen as $s$ or $p$ by the use of HWP2 and HWP3. The required optical components in these different situations are summarized in Table $\underline{1}$.

\section{RESULTS}

The curves in Figs. $3(\mathrm{a})-3(\mathrm{~d})$ show the results of obliqueincidence interferometric measurements. The curves (a) and (b) correspond to the real $\left(\rho_{s}\right)$ and imaginary $\left(\delta \phi_{s}\right)$ parts of the relative reflectance change for $s$-polarized probe light, whereas (c) and (d) correspond to those for $p$-polarized probe light $\left(\rho_{p}\right.$ and $\left.\delta \phi_{p}\right)$.

Consider first Figs. 3(a) and 3(c), corresponding to $\rho_{s}$ and $\rho_{p}$, respectively. A sharp peak is observed at zero delay time $(t=0)$. This results from the electron temperature rise caused by the absorption of the pump photon energy and from the subsequent energy transfer to the lattice (see Section 5). After this sharp peak, a gradual decrease is observed, related to the cooling of the sample. Upon this slowly decaying background, an echo is observed at $120 \mathrm{ps}$. This is caused by a longitudinal coherent phonon pulse returning after reflection from the film-substrate interface. The echo consists of a central peak and two small dips on either side. This is a typical shape for echoes arising from the photoelastic effect in opaque samples. Half of this shape is also observed after zero delay time owing to the departure of the coherent phonon pulse from the film surface after thermoelastic generation. A second, smaller echo is also resolved at $240 \mathrm{ps}$.

In Figs. 3(b) and 3(d), corresponding to $\delta \phi_{s}$ and $\delta \phi_{p}$, respectively, 3 echoes are clearly observed at times determined by the sound velocity $v_{l}=5160 \mathrm{~ms}^{-1}$ [44] and thickness (estimated to be $d=313 \mathrm{~nm}$ ) of the tungsten film. As shown in Eqs. (15) and (16), the terms contributing from the photoelastic effect are similar to a spatial Fourier transform of the strain field, and exhibit a high sensitivity to the wavenumber components of the strain field $\eta_{z z}(z, t)$ near $\left|k_{\text {strain }}\right| \simeq 2 k^{\prime \prime}$ (where $k_{\text {strain }}$ is the acoustic wavenumber). This selectivity is essentially the same as that involved in the detection of Brillouin oscillations in transparent media. In the curves (a) and (c) for $\rho_{s}$ and $\rho_{p}$, such frequency components are involved in the first echo, but not much in higher-order echoes because of frequency-dependent ultrasonic attenuation. This also implies that the second and third echoes in curves (b) and (d) for $\delta \phi_{s}$ and $\delta \phi_{p}$ are less affected by the photoelastic contribution than the first peak is, and that the decrease of the peak height in the curves (b) and (d) approximately follows the decrease of the overall strain pulse height.

The data of Figs. $3(\mathrm{a})-3(\mathrm{~d})$ are in overall agreement with previous laser-picosecond-ultrasonics based investigations on tungsten films $[\underline{7,}, \underline{8}, \underline{11}, \underline{17}, \underline{29}, \underline{41}]$; the contribution to the echo shape from the photoelastic effect depends strongly on the probe wavelength [11] and probe incident angle [45]. The latter dependence is evident in Eqs. (15) and (16).

Our direct displacement measurement consists of three steps. The first step is an oblique-incidence reflectivity change measurement with $s$ - or $p$-polarized light corresponding to the

Table 1. Required Optical Components for Different Interferometric Measurements ${ }^{a}$

\begin{tabular}{|c|c|c|c|c|}
\hline Functionality & QWP1 & POL2 & QWP2 & POL3 \\
\hline Cancellation of photoelastic contribution & 。 & $\circ$ & - & $\mathrm{H}$ \\
\hline Direct displacement measurement & $\circ$ & $\circ$ & $\circ$ & $\circ$ \\
\hline Oblique incidence interferometer & - & - & $\circ$ & $\circ$ \\
\hline Simple reflectivity measurement & - & - & - & $\mathrm{H}$ \\
\hline
\end{tabular}




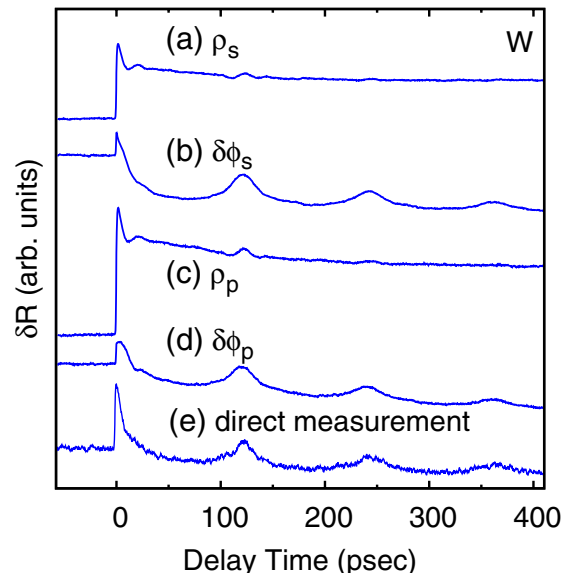

Fig. 3. (a)-(d) Oblique-incidence interferometric results for the W/crown-glass sample. (a) Real and (b) imaginary parts with $s$ polarized probe light. (c) Real and (d) imaginary parts with $p$-polarized probe light. (e) Result of direct displacement measurement.

data for $\rho_{s}$ or $\rho_{p}$ in Fig. 3. This step confirms the correct overlap of pump and probe spot positions; in the following two steps, the spot positions are kept unchanged. The second step is an interferometric measurement with a mixture of $s$ - and $p$-polarized light with appropriate amplitude ratio and phase difference to cancel the photoelastic contribution. The third step is the direct measurement of the surface displacement with the incorporation of reference light in the interferometric setup.

The results corresponding to these steps are shown in Fig. 4. The curve (a) is the result of the first step, i.e., the reflectivity change with $p$-polarized light, or $\rho_{p}$. The curve (b) is the result of the second step: by adjusting the intensity ratio (HWP2) and the phase retardation (POL2 and HWP3) between the $s$ - and $p$-polarized components of the probe light, almost perfect cancellation of the photoelastic effect is achieved, as shown by the disappearance of the echo at 120 ps [46]. The curve (c) is the result of the third step. The probe light reflected from the sample in the second step does not show an intensity variation corresponding to an echo, but it still contains a corresponding phase variation caused by the surface displacement. By mixing reference light with a $\pi / 2$ phase retardation (or advance) with the reflected probe light, this phase variation is converted to an intensity variation proportional to the surface displacement. When the phase of the reference light is shifted by $\pi$, one expects the polarity of the detected light intensity to be flipped. This is indeed the case for curve (d). These results corroborate the observation of surface displacements without contamination by the photoelastic effect. Unlike the photoelastic contribution, which has a nonuniform sensitivity for different acoustic wavenumbers (or corresponding frequencies), this direct displacement measurement has a flat frequency response up to terahertz acoustic frequencies ( the inverse of the optical pulse duration).

A $z$-propagating strain field in a dispersionless semi-infinite medium with a free surface at $z=0$ can be expressed as

$$
\eta(z, t)=f(z-v t)+f(z+v t)
$$

where $v$ is the sound velocity. The free boundary condition at the surface implies $f(\xi)=-f(-\xi)$. The surface displacement is then given by

$$
u_{z}(0, t)=\int_{+\infty}^{0}\left\{f\left(z^{\prime}-v t\right)+f\left(z^{\prime}+v t\right)\right\} \mathrm{d} z^{\prime}
$$

By taking the temporal partial derivative of Eq. (23), the strain waveform $f(\xi)$ is given by [3]

$$
\left.\frac{\partial u_{z}}{\partial t}\right|_{z=0}=2 v f(v t) .
$$

In other words, by monitoring the temporal derivative of the surface displacement, one can extract the coherent phonon strain pulse shape. The corresponding experimental curve is shown in Fig. 4(e). The strain pulse width (at FWHM of $\left.\left|\partial u_{z} / \partial t\right|\right)$ is $\sim 40 \mathrm{ps}$, and is much broader than the central peak of the echo observed in Fig. 4(a) by means of the photoelastic effect.

We reproduce the results of the direct displacement measurement [corresponding to the upper curve in Fig. 4(c)] in Fig. 3(e) for comparison purposes. In tungsten, it turns out that the photoelastic contribution to the probe optical phase variation is, for $s$ - and $p$-polarizations, $\sim 7 \%$ and $\sim 20 \%$, respectively, of the surface displacement contribution, and so the curves in Figs. 3(b), 3(d), and 3(e) are similar. However, the top of the first echo in Fig. $3(\mathrm{e})$ is somewhat sharper than the corresponding features in the curves (b) and (d). This shows that the curves (b) and (d) are still affected by the photoelastic contribution. This will be shown in detail in the next section.

\section{DISCUSSION}

In this section, we first explain a model that can account for the strain pulse shape and at the same time can retrieve

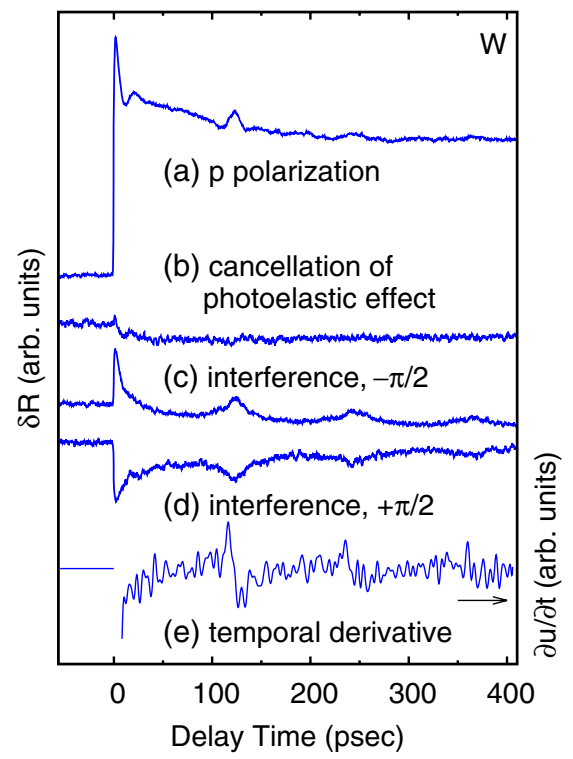

Fig. 4. Measured probe beam intensity variations for the W/crownglass sample. (a) Oblique-incidence reflectivity change measurement with $p$-polarized probe light. (b) Use of an appropriate mixture of $p$ and $s$-polarized probe light in an interferometric setup cancels the photoelastic contribution. (c) Introduction of reference light with a $\pi / 2$ phase retardation in this setup converts phase modulations from surface displacements into intensity variations. (d) A flip of polarity is observed by the use of reference light with a $\pi$ phase shift compared to that used in (c). (e) Measured temporal derivative of the surface displacement. 
physical properties of the tungsten film. We include the ultrafast dynamics of the photoexcited electrons, the generation and propagation of acoustic waves, and the modulation of the reflected probe light through the photoelastic effect, the surface displacement, and the thermo-optic effect.

For the present experimental geometry, the thermoelastic generation and acoustic propagation can be regarded as a one-dimensional problem involving the coordinate $z$. For thermoelastic expansion in an isotropic medium, only longitudinal acoustic waves are directly generated; in our onedimensional treatment, mode conversion to shear waves is also precluded. The acoustic wave equation is then given by

$$
\rho \frac{\partial^{2} u_{z}}{\partial t^{2}}=\frac{\partial \sigma_{z z}}{\partial z}=c_{11} \frac{\partial^{2} u_{z}}{\partial z^{2}}-\left(c_{11}+2 c_{12}\right) \beta \frac{\partial \Delta T}{\partial z},
$$

where $\rho$ is the mass density, $\sigma_{i j}$ are stress tensor components, $c_{I J}$ are elastic stiffness tensor components, $\beta$ is the linear thermal expansion coefficient, and $\Delta T$ is the temperature rise.

The spatial extent of the generated strain pulse, as shown in curve (c) in Fig. 4, is significantly larger, $200 \mathrm{~nm}$ (equivalent to $\sim 40 \mathrm{ps})$, than the pump optical penetration depth $(\sim 30 \mathrm{~nm})$ because of ultrafast electron diffusion during generation. This process can be described by the two-temperature model, in which the electron and lattice systems are considered to be in quasi-independent thermal equilibrium characterized by the temperatures $T_{e}$ and $T_{l}$, respectively, but interacting in a nonlinear fashion through the electron-phonon interaction [47]:

$$
\begin{aligned}
C_{e} \frac{\partial T_{e}}{\partial t} & =\frac{\partial}{\partial z}\left(\kappa \frac{\partial T_{e}}{\partial z}\right)-g\left(T_{e}-T_{l}\right)+S(z, t), \\
C_{l} \frac{\partial T_{l}}{\partial t} & =g\left(T_{e}-T_{l}\right),
\end{aligned}
$$

where $C_{e}=\gamma T_{e}$ and $C_{l}$ are the volume specific heats of the electron and lattice systems, respectively, $g$ is the electronphonon coupling constant, and $\kappa=\kappa_{0} T_{e} / T_{l}$ is the thermal conductivity, where $\kappa_{0}$ is the value of $\kappa$ when the electron and lattice systems are in equilibrium $[48,49]$. The lattice heat diffusion is much smaller than the electron heat diffusion in metals, and is ignored here. $S(z, t)$ is the deposited energy per unit time per unit volume by the absorption of a pump light pulse. At the film surface and the film/substrate interface, there should be no electron flow. This corresponds to the boundary condition $\kappa\left(\partial T_{e} / \partial z\right)=0$. Equation (26) is solved numerically by a finite difference time domain method to obtain the spatiotemporal evolution of $T_{e}$ and $T_{l}$. Immediately after the excitation by a light pulse with a temporal width shorter than $1 \mathrm{ps}, T_{e}$ becomes in our case higher by several tens of $\mathrm{K}$, whereas $T_{l}$ stays near room temperature. The electron energy is then transferred to the lattice. Since the electrons diffuse during this thermalization process, the depth of the region with raised $T_{l}$ is generally larger than the optical penetration depth. In general, the spatial distributions of $T_{e}$ and $T_{l}$ at a particular time (e.g., several ps) after pump pulse absorption are broader for metals with smaller $g$ or larger $\kappa$. The spatiotemporal evolution of the strain field $\eta_{z z}(z, t)$ can be calculated from Eq. (25) with a knowledge of $\Delta T$, defined here as the variation of $T_{l}$ from its initial value. In this theoretical simulation, the maximum transient lattice temperature rise is about $1 \mathrm{~K}$ [50].
In Fig. 3, the ratio between the heights of the first and second echoes for $\rho_{s}$ and $\rho_{p}$ is about 0.3 , whereas that for $\delta \phi_{s}$ and $\delta \phi_{p}$ is about 0.6. As discussed in Section $\underline{4}, \rho_{s}$ and $\rho_{p}$ arise from the photoelastic effect, and have a sensitivity characterized by a nonuniform acoustic frequency spectrum, whereas $\delta \phi_{s}$ and $\delta \phi_{p}$ are mostly governed by the surface displacement, and have a nearly frequency-independent sensitivity. The observed difference in the echo height ratio points to the presence of a frequency-dependent ultrasonic absorption coefficient $\alpha$. We assume a variation in the empirical form

$$
\alpha=a+b f^{2}
$$

where $f=\omega / 2 \pi$, and $a$ and $b$ are constant coefficients. The quadratic frequency absorption coefficient $b$ may contain contributions from viscoelasticity $[\underline{51}, \underline{52}]$, whereas the frequency-independent absorption coefficient $a$ can arise from deviations of the acoustic reflection coefficient at the W/crown-glass interface from the assumed literature value. The values of $a$ and $b$ can be obtained by comparison of the Fourier amplitude of the first, second, and third echoes [53]. This attenuation is also included in the simulation of the propagation of the coherent phonon pulses. The detailed procedure is described elsewhere [54].

Once $\eta_{z z}(z, t)$ is obtained, Eqs. (15) and (16) can be used to calculate the optical transient reflectance change. But, as shown in Fig. 4(b), there is a residual negative reflectivity step even when the photoelastic contribution is effectively cancelled. To explain the presence of this step, we consider a permittivity modulation by the temperature variation (i.e., the thermo-optic effect) in addition to that by the photoelastic effect. The delay time in question is greater than a few picoseconds, at a time when the electrons can be regarded as thermalized with the lattice. This thermo-optic effect is included by replacing $\varepsilon_{\mathrm{pe}}$ in Eq. (1ㅏ) with $[\underline{17}, \underline{55}]$

$$
\Delta \varepsilon_{i j}=P_{\mathrm{to}} \delta_{i j} \Delta T+P_{i j k l} \eta_{k l} \text {. }
$$

Here, $P_{\text {to }}$ and $P_{i j k l}$ are complex thermo-optic coefficients and photoelastic tensor components, respectively. The second term in this equation is the photoelastic contribution, $\varepsilon_{\mathrm{pe}}$, obtained from Eq. (12) for pure longitudinal waves $\left(\eta_{i j}=0\right.$ except $\eta_{z z}$ ), and is not an isotropic second-rank tensor, whereas the first term, the thermo-optic contribution, is. Because of the mixed isotropic/anisotropic nature of these terms, the reflectance change arising from the thermo-optic contribution does not in general cancel when that from the photoelastic contribution does.

In Fig. 5, the experimental intensity variation obtained from curve (c) in Fig. $\underline{4}$ is fitted in the region from 80 to 400 ps using the parameters shown in Table 2 . The temporal range from 0 to $80 \mathrm{ps}$ is not well fitted partly because of the excited electron contribution, which is not included here. For the permittivity at the pump light wavelength $(830 \mathrm{~nm})$, the value obtained by ellipsometry measurement on our sample is adopted rather than the literature value [44] of $4.1+19.4 i$ [60]. The thermo-optic contribution and the surface displacement contribution are also shown separately in Figs. 5(c) and 5(d). The slow background decay in curve (a) can be attributed to the (equilibrated electron and lattice) temperature variation. The surface displacement contribution gives the echos as well as the flat (in fact, step-like) negative background which is 


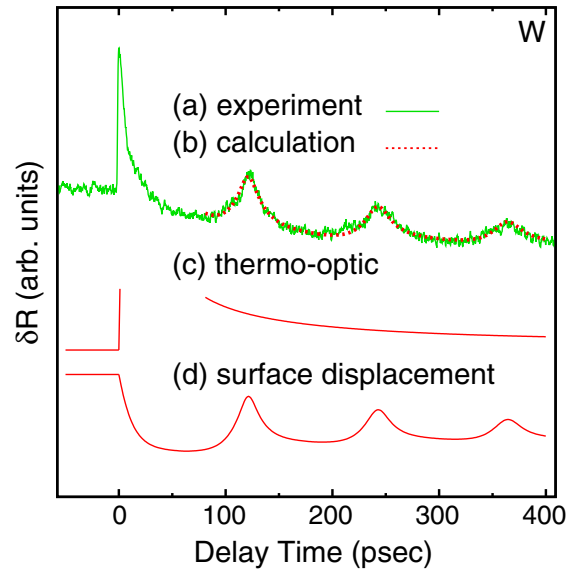

Fig. 5. (a) Experimental intensity variation from the interferometric setup with a $\pi / 2$ phase retardation (solid line) and (b) corresponding theoretical variation (dashed line) for the W/crown-glass sample. Contributions to (b) are separately plotted as (c) the thermo-optic contribution, and (d) the surface displacement contribution.

caused by the (quasi-) static strain distribution arising from thermal expansion. The numerical value of the surface displacement corresponding to the first echo is $\sim 0.6 \mathrm{pm}$, in reasonable agreement with the predictions of our model $(0.4 \mathrm{pm})$.

In particular, the fitting yields a value for the electronphonon coupling constant $g$, as shown with other parameters in Table 2. We obtain a possible range of $g$ values from $1 \times 10^{17}$ to $1 \times 10^{18} \mathrm{Wm}^{-3} \mathrm{~K}^{-1}$, with a least-squares optimal value at $g=3 \times 10^{17} \mathrm{Wm}^{-3} \mathrm{~K}^{-1}$. This value is in good agreement with the values in [61] $\left(g \simeq 5 \times 10^{17}\right.$ to $1 \times 10^{18} \mathrm{Wm}^{-3} \mathrm{~K}^{-1}$ obtained from transient reflectivity changes), [7,62] $[g \simeq(2.6 \pm 0.39) \times$ $10^{17} \mathrm{Wm}^{-3} \mathrm{~K}^{-1}$ from the transient reflectivity changes], and [17] $\left(g=1.0 \times 10^{17} \mathrm{Wm}^{-3} \mathrm{~K}^{-1}\right.$ from optical interferometric measurements of acoustic pulse shapes). These various values for $g$ might arise because of differences in sample

Table 2. Parameters Used for the Theoretical Simulation of the Tungsten Film ${ }^{a}$

\begin{tabular}{|c|c|}
\hline Thermal conductivity [ $\underline{56}$ ], $\kappa_{0}\left(\mathrm{Wm}^{-1} \mathrm{~K}^{-1}\right)$ & $1.78 \times 10^{2}$ \\
\hline Lattice specific heat [5]], $C_{l}\left(\mathrm{Jm}^{-3} \mathrm{~K}^{-1}\right)$ & $2.53 \times 10^{6}$ \\
\hline Constant for electron specific heat [58] & \\
\hline$\gamma\left(\mathrm{Jm}^{-3} \mathrm{~K}^{-2}\right)$ & $1.37 \times 10^{2}$ \\
\hline Electron-phonon coupling constant* & \\
\hline $\begin{array}{l}g\left(\mathrm{Wm}^{-3} \mathrm{~K}^{-1}\right) \\
\text { Mass density, } \rho\left(\mathrm{kgm}^{-3}\right)\end{array}$ & $3 \times 10^{17}$ \\
\hline for $\mathrm{W}[44]$ & $1.93 \times 10^{4}$ \\
\hline for crown glass [59] & $2.24 \times 10^{3}$ \\
\hline Electrical permittivity, $\varepsilon_{1}$ & \\
\hline for $\mathrm{W}$ at $830 \mathrm{~nm}$ & $4.6+5.5 i$ \\
\hline Thermal expansion coefficient [44], $\beta\left(\mathrm{K}^{-1}\right)$ & $4.5 \times 10^{-6}$ \\
\hline Elastic stiffness $(\mathrm{Pa})$ & \\
\hline$c_{11}$ for polycrystalline $\mathrm{W}[\underline{44}]$ & $5.13 \times 10^{11}$ \\
\hline$c_{44}$ & $1.53 \times 10^{11}$ \\
\hline$c_{11}$ for crown glass [59] & $5.82 \times 10^{10}$ \\
\hline$c_{44}$ & $1.81 \times 10^{10}$ \\
\hline Film thickness, ${ }^{*} d(\mathrm{~nm})$ & $3.13 \times 10^{2}$ \\
\hline Ultrasonic absorption coefficient* & \\
\hline$a\left(\mathrm{~m}^{-1}\right)$ & $3 \times 10^{5}$ \\
\hline$b\left(\mathrm{~m}^{-1} \mathrm{GHz}^{-2}\right)$ & $4 \times 10^{2}$ \\
\hline Thermo-optic coefficient ${ }^{*}, P_{\text {to }}\left(\mathrm{K}^{-1}\right)$ & $(-2.0-0.4 i) \times 10^{-3}$ \\
\hline
\end{tabular}

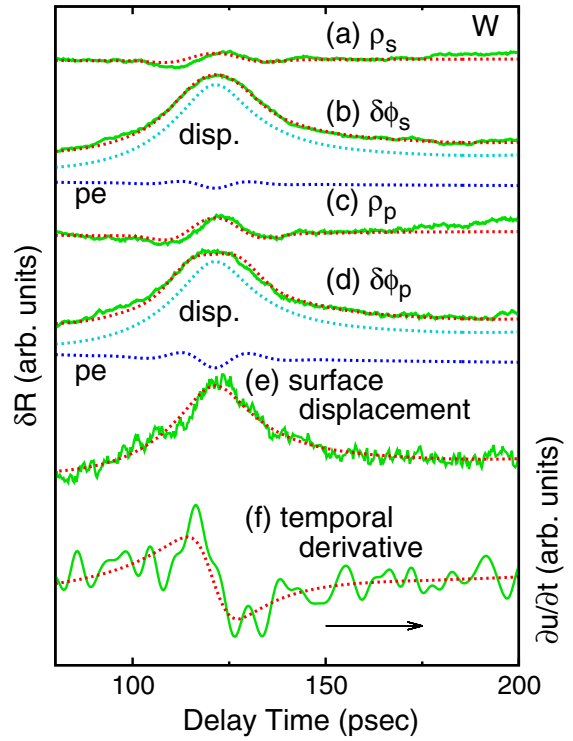

Fig. 6. First-echo experimental results (solid lines) and theory (dashed lines) for the W/crown-glass sample. (a) Real and (b) imaginary part of the relative reflectance change with $s$-polarized probe light. (c) Real and (d) imaginary parts with $p$-polarized probe light. (e) Surface displacement variation. (f) Temporal derivative of the surface displacement. For (b) and (d), the photoelastic (pe) and surface displacement (disp.) contributions are also shown, shifted for clarity. In (a)-(e), the thermo-optic background variations are subtracted.

purity or microstructure. Further studies, for example with varying film thickness or preparation method, would help elucidate this point.

As a check on our theoretical approach, we now attempt to reproduce all the experimental data for the first echoes by the use of Eqs. (15) and (16). Figures 6(a)-6(d) show the results for amplitude and phase for the first echo, with thermo-optic background subtracted, from experiment (solid lines) and from theory (dotted lines), which exhibit very good agreement overall. In (b) and (d) for $\delta \phi_{s}$ and $\delta \phi_{p}$, respectively, the photoelastic (pe) and surface displacement (disp.) contributions are shown below their sum. Table 3 shows the optical and photoelastic parameters obtained by least-squares fitting [63]. In (e) and (f), the surface displacement with thermo-optic background subtracted and its temporal derivative (i.e., the shape of the strain pulse propagating inside the film) are also, respectively, shown, and exhibit reasonable agreement between experiment and theory within the noise level.

Figure 7 shows the first-echo frequency spectrum obtained experimentally (solid line) and theoretically (dashed line) from the displacement variation [64]. Good agreement is obtained, with frequency components significant up to $\sim 50 \mathrm{GHz}$ (limited by electron diffusion).

\section{Table 3. Parameters Used for the Theoretical Simulations for Oblique-Incidence Interferometric Measurements ${ }^{a}$}

Electrical permittivity, $\varepsilon_{1}$

for $\mathrm{W}$ at $415 \mathrm{~nm}$

Photoelastic constants for $\mathrm{W}$ at $415 \mathrm{~nm}$

$P_{11}$

$$
3.8+4.4 i
$$

$P_{12}$

${ }^{a}$ Values are obtained by the fitting. 


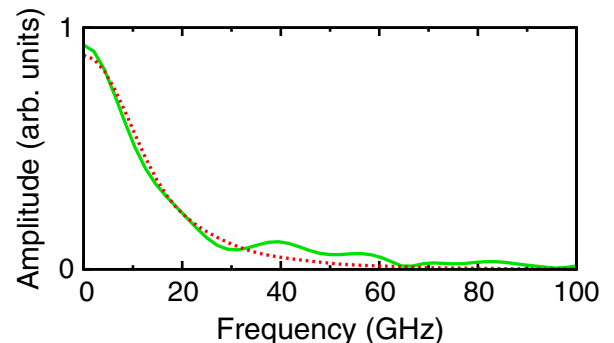

Fig. 7. Frequency spectrum for the first echo obtained experimentally (solid line) and theoretically (dashed line) from the surface displacement for the W/crown-glass sample.

\section{CONCLUSIONS}

In conclusion, we have demonstrated an oblique-incidence common-path interferometric method based on ellipsometry to directly observe the surface displacement caused by propagating picosecond longitudinal coherent phonon pulses in a metal film, thus allowing the strain pulse shape inside the film to be extracted. The measurement, free from the photoelastic effect, is applied to a thin tungsten film. We interpret the observed strain pulse shape by a model of the ultrafast dynamics of the excited electrons, and derive an estimate for the electron-phonon coupling constant. A thermo-optic contribution to the reflectance is also identified. Our technique can reliably determine gigahertz acoustic strain pulse shapes because of the flat acoustic sensitivity spectrum up to terahertz frequencies, and should be applicable to other types of samples, such as bulk semiconductors or quantum heterostructures.

\section{APPENDIX A: ARBITRARY PHASE RETARDER}

The combination of a quarter-wave plate (QWP) and a polarizer can be used to interfere $s$ - and $p$-polarized light components with an arbitrary phase retardation. The principle of this method is briefly explained in this Appendix.

Consider light propagating along the $Z$ axis in Fig. 8(a). We assume that the incident light (complex) amplitude at $\bar{Z}=0$ is given by

$$
\mathbf{E}=\left(\begin{array}{c}
E_{X} \\
E_{Y}
\end{array}\right)
$$

A QWP with its fast and slow axes oriented $45^{\circ}$ to the horizontal plane, as shown in Fig. $\underline{8(b)}$, has a Jones matrix

$$
\mathrm{QWP}_{45}=\frac{1}{2}\left(\begin{array}{cc}
1+i & -1+i \\
-1+i & 1+i
\end{array}\right)
$$

(a)

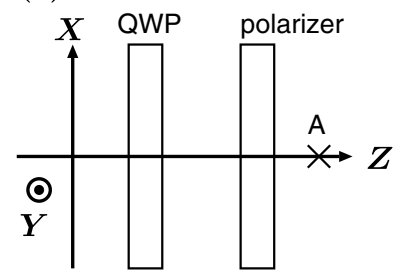

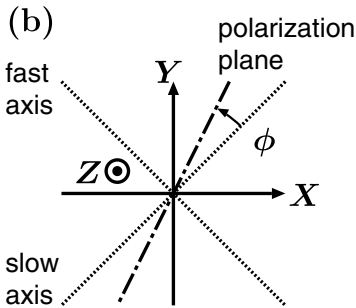

Fig. 8. (a) Combination of a quarter-wave plate (QWP) and a polarizer. (b) Axis definitions looking from downstream.
A polarizer with its polarization plane rotated by $\pi / 4+\phi$ from the $X$ axis is placed behind the QWP. Then the amplitude of the electric field along the polarization plane at the point $\mathrm{A}$ is given by

$$
\begin{aligned}
E_{A} & =\left(\cos \left(\frac{\pi}{4}+\phi\right) \sin \left(\frac{\pi}{4}+\phi\right)\right) \mathrm{QWP}_{45} \mathbf{E} \\
& =\frac{i}{\sqrt{2}}\left(E_{X} \exp (+i \phi)+E_{Y} \exp (-i \phi)\right) .
\end{aligned}
$$

One can interpret this by thinking of $E_{X}$ as retarded by $2 \phi$ and then added to $E_{Y}$. In this way, it is possible to interfere two orthogonal polarization components with arbitrary phase retardation by a single adjustment of the polarizer angle $\phi$.

\section{ACKNOWLEDGMENTS}

This work is partially supported by a Grant-in-Aid for Scientific Research from the Ministry of Education, Science, Sports, and Culture of Japan.

\section{REFERENCES AND NOTES}

1. C. Thomsen, J. Strait, Z. Vardeny, H. J. Maris, J. Tauc, and J. J. Hauser, "Coherent phonon generation and detection by picosecond light pulses,” Phys. Rev. Lett. 53, 989-992 (1984).

2. C. Thomsen, H. T. Grahn, H. J. Maris, and J. Tauc, "Surface generation and detection of phonons by picosecond light pulses," Phys. Rev. B 34, 4129-4138 (1986).

3. O. B. Wright and K. Kawashima, "Coherent phonon detection from ultrafast surface vibrations," Phys. Rev. Lett. 69, 16681671 (1992).

4. G. Tas and H. J. Maris, "Electron diffusion in metals studied by picosecond ultrasonics," Phys. Rev. B 49, 15046-15054 (1994).

5. O. B. Wright, "Ultrafast nonequilibrium stress generation in gold and silver,” Phys. Rev. B 49, 9985-9988 (1994).

6. B. Perrin, B. Bonello, J.-C. Jeannet, and E. Romatet, "Interferometric detection of hypersound waves in modulated structures," Prog. Nat. Sci. S6, S444-S448 (1996).

7. J. L. Hostetler, A. N. Smith, and P. M. Norris, "Thin-film thermal conductivity and thickness measurements using picosecond ultrasonics," Microscale Thermophys. Eng. 1, 237-244 (1997).

8. C. J. K. Richardson, M. J. Ehrlich, and J. W. Wagner, "Interferometric detection of ultrafast thermoelastic transients in thin films: theory with supporting experiment," J. Opt. Soc. Am. B 16, 1007-1015 (1999).

9. D. H. Hurley and O. B. Wright, "Detection of ultrafast phenomena by use of a modified Sagnac interferometer," Opt. Lett. 24, 1305-1307 (1999).

10. C. K. Sun, J. C. Liang, and X. Y. Yu, "Coherent acoustic phonon oscillations in semiconductor multiple quantum wells with piezoelectric fields," Phys. Rev. Lett. 84, 179-182 (2000).

11. A. Devos and C. Lerouge, "Evidence of laser-wavelength effect in picosecond ultrasonics: possible connection with interband transitions," Phys. Rev. Lett. 86, 2669-2672 (2001).

12. O. B. Wright, B. Perrin, O. Matsuda, and V. E. Gusev, "Ultrafast carrier diffusion in gas probed with picosecond acoustic pulses," Phys. Rev. B 64, 081202(R) (2001).

13. T. Saito, O. Matsuda, and O. B. Wright, "Picosecond acoustic phonon pulse generation in nickel and chromium," Phys. Rev. B 67, 205421 (2003).

14. O. Matsuda, T. Tachizaki, T. Fukui, J. J. Baumberg, and O. B. Wright, "Acoustic phonon generation and detection in GaAs $/ \mathrm{Al}_{0.3} \mathrm{Ga}_{0.7} \mathrm{As}$ quantum wells with picosecond laser pulses," Phys. Rev. B 71, 115330 (2005).

15. A. Huynh, N. D. Lanzillotti-Kimura, B. Jusserand, B. Perrin, A. Fainstein, M. F. Pascual-Winter, E. Peronne, and A. Lemaître, "Subterahertz phonon dynamics in acoustic nanocavities," Phys. Rev. Lett. 97, 115502 (2006).

16. A. V. Akimov, A. V. Scherbakov, D. R. Yakovlev, C. T. Foxon, and M. Bayer, "Ultrafast band-gap shift induced by a strain pulse in 
semiconductor heterostructures," Phys. Rev. Lett. 97, 037401 (2006).

17. T. Dehoux, M. Perton, N. Chigarev, C. Rossignol, J. M. Rampnoux, and B. Audoin, "Effect of laser pulse duration in picosecond ultrasonics," J. Appl. Phys. 100, 064318 (2006).

18. M. F. P. Winter, G. Rozas, A. Fainstein, B. Jusserand, B. Perrin, A. Huynh, P. O. Vaccaro, and S. Saravanan, "Selective optical generation of coherent acoustic nanocavity modes," Phys. Rev. Lett. 98, 265501 (2007).

19. K.-H. Lin, C.-M. Lai, C.-C. Pan, J.-I. Chyi, J.-W. Shi, S.-Z. Sun, C.-F. Chang, and C.-K. Sun, "Spatial manipulation of nanoacoustic waves with nanoscale spot sizes," Nature Nanotech. 2, 704708 (2007).

20. C. Rossignol, N. Chigarev, M. Ducousso, B. Audoin, G. Forget, F. Guillemot, and M. C. Durrieu, "In vitro picosecond ultrasonic in a single cell," Appl. Phys. Lett. 93, 123901 (2008).

21. O. Matsuda, O. B. Wright, D. H. Hurley, V. Gusev, and K. Shimizu, "Coherent shear phonon generation and detection with picosecond laser acoustics," Phys. Rev. B 77, 224110 (2008).

22. O. B. Wright, B. Perrin, O. Matsuda, and V. E. Gusev, "Optical excitation and detection of picosecond acoustic pulses in liquid mercury," Phys. Rev. B 78, 024303 (2008).

23. P. Babilotte, P. Ruello, G. Vaudel, T. Pezeril, D. Mounier, J. M. Breteau, and V. Gusev, "Picosecond acoustics in p-doped piezoelectric semiconductors,” Appl. Phys. Lett. 97, 174103 (2010).

24. H. Ogi, A. Yamamoto, K. Kondou, K. Nakano, K. Morita, N. Nakamura, T. Ono, and M. Hirao, "Significant softening of copper nanowires during electromigration studied by picosecond ultrasound spectroscopy," Phys. Rev. B 82, 155436 (2010).

25. C. Klieber, E. Peronne, K. Katayama, J. Choi, M. Yamaguchi, T. Pezeril, and K. A. Nelson, "Narrow-band acoustic attenuation measurements in vitreous silica at frequencies between 20 and $400 \mathrm{GHz}$," Appl. Phys. Lett. 98, 211908 (2011).

26. E. Pontecorvo, M. Ortolani, D. Polli, M. Ferretti, G. Ruocco, G. Cerullo, and T. Scopigno, "Visualizing coherent phonon propagation in the $100 \mathrm{GHz}$ range: a broadband picosecond acoustics approach," Appl. Phys. Lett. 98, 100901 (2011).

27. Y.-C. Wen, K.-J. Wang, H.-H. Chang, J.-Y. Luo, C.-C. Shen, H.-L. Liu, C.-K. Sun, M.-J. Wang, and M.-K. Wu, "Gap opening and orbital modification of superconducting FeSe above the structural distortion," Phys. Rev. Lett. 108, 267002 (2012).

28. J.-W. Kim, M. Vomir, and J.-Y. Bigot, "Ultrafast magnetoacoustics in nickel films," Phys. Rev. Lett. 109, 166601 (2012).

29 . N. Chigarev, C. Rossignol, and B. Audoin, "Surface displacement measured by beam distortion detection technique: application to picosecond ultrasonics," Rev. Sci. Instrum. 77, 114901 (2006)

30. O. Matsuda and O. B. Wright, "Laser picosecond acoustics with oblique probe light incidence," Rev. Sci. Instrum. 74, 895-897 (2003).

31. O. Matsuda, K. Aoki, T. Tachizaki, and O. Wright, "Direct measurement of ultrafast surface displacement in laser picosecond acoustics," J. Phys. IV 125, 361-363 (2005).

32. R. M. A. Azzam and N. M. Bashara, Ellipsometry and Polarized Light (North-Holland, 1977).

33. J. A. Bolger, A. E. Paul, and A. L. Smirl, "Ultrafast ellipsometry of coherent processes and exciton-exciton interactions in quantum wells at negative delays," Phys. Rev. B 54, 11666-11671 (1996).

34. C. A. Bolme, S. D. McGrane, D. S. Moore, and D. J. Funk, "Single shot measurements of laser driven shock waves using ultrafast dynamic ellipsometry," J. Appl. Phys. 102, 033513 (2007).

35. L. R. Watkins, "Interferometric ellipsometer," Appl. Opt. 47, 2998-3001 (2008).

36. D. Mounier, E. Morozov, P. Ruello, J. M. Breteau, P. Picart, and V. Gusev, "Detection of shear picosecond acoustic pulses by transient femtosecond polarimetry," Eur. J. Phys. Special Topics 153, 243-246 (2008)

37. C.-K. Min, D. G. Cahill, and S. Granick, "Time-resolved ellipsometry for studies of heat transfer at liquid/solid and gas/solid interfaces," Rev. Sci. Instrum. 81, 074902 (2010).

38. O. Matsuda and O. B. Wright, "Reflection and transmission of light in multilayers perturbed by picosecond strain pulse propagation,” J. Opt. Soc. Am. B 19, 3028-3041 (2002).
39. A. A. Maradudin and D. L. Mills, "Scattering and absorption of electromagnetic radiation by a semi-infinite medium in the presence of surface roughness," Phys. Rev. B 11, 1392-1415 (1975).

40. W is optically isotropic because it crystallizes in the cubic (bcc) phase. In addition, the elastic constants for tungsten single crystal are $c_{11}=502, c_{44}=152$, and $c_{12}=199 \mathrm{GPa}$ [59], and coincidentally obey the relation $c_{11}-c_{12} \simeq 2 c_{44}$, so that (independent of the film microstructure) the film is effectively elastically isotropic.

41. O. B. Wright and K. Kawashima, "Ultrasonic detection from picosecond surface vibrations: application to interfacial layer detection," Jpn. J. Appl. Phys. 32, 2452-2454 (1993).

42. The surface roughness contributes to the frequency-dependent ultrasonic absorption (see [22]), but we estimate its effect to be small over the frequency spectrum of the ultrasonic pulse associated with the first echo. The roughness of the W/crown-glass interface is $\sim 5 \mathrm{~nm}$, and its effect can also be neglected.

43. T. Tachizaki, T. Muroya, O. Matsuda, Y. Sugawara, D. H. Hurley, and O. B. Wright, "Scanning ultrafast sagnac interferometry for imaging two-dimensional surface wave propagation," Rev. Sci. Instrum. 77, 043713 (2006).

44. D. R. Lide, ed., CRC Handbook of Chemistry and Physics, 85th ed. (CRC Press, 2004)

45. M. Tomoda, O. Matsuda, and O. B. Wright, "Tomographic reconstruction of picosecond acoustic strain propagation," Appl. Phys. Lett. 90, 041114 (2007).

46. Some residual photoelastic effect that was not cancelled is, however, still visible near $t=0$. This level of small remnant should not significantly affect the shape of the final extracted surface displacement variations.

47. S. I. Anisimov, B. L. Kapeliovich, and T. L. Perel'man, "Emission of electrons from the surface of metals induced by ultrashort laser pulses," Sov. Phys. JETP 39, 375-377 (1974).

48. P. B. Corkum, F. Brunel, N. K. Sherman, and T. Srinivasan-Rao, "Thermal response of metals to ultrashort-pulse laser excitation," Phys. Rev. Lett. 61, 2886-2889 (1988).

49. V. E. Gusev and O. B. Wright, "Ultrafast nonequilibrium dynamics of electrons in metals," Phys. Rev. B 57, 2878-2888 (1998).

50. The simulation involves a nonlinear response: when the excitation energy flux is halved, for example, the maximum changes in $T_{e}$ and $T_{l}$ are, respectively, $1.4 \%$ and $0.3 \%$ greater than the corresponding halved values. This nonlinear response is below the detection limit of the measurement here.

51. R. T. Beyer and S. V. Letcher, Physical Ultrasonics (Academic, 1969), Chap. 10, pp. 325-358.

52. H. Klein and O. Weis, "Absorption heat of GHz sound in polycrystalline metal films detected by multiple-beam interferometry and second-sound emission," J. Low Temp. Phys. 94, 567-583 (1994).

53. We adopted $b=4 \times 10^{2} \mathrm{~m}^{-1} \mathrm{GHz}^{-2}$, which gives optimal agreement in the fitting of the surface displacement data, although other $b$ differing by values of the same order also gave acceptable fits. On the other hand, $a$ is more accurately obtained as $a=(3.0 \pm 0.5) \times 10^{5} \mathrm{~m}^{-1}$ for the chosen value of $b$. Our expression for $b f^{2}$ is consistent with values of $\alpha$ found at $1 \mathrm{GHz}$ in $\mathrm{W}$ $\left(\alpha=100-600 \mathrm{~m}^{-1}\right.$, see [65-67]).

54. S. Kashiwada, O. Matsuda, J. J. Baumberg, R. L. Voti, and O. B. Wright, "In situ monitoring of the growth of ice films by laser picosecond acoustics," J. Appl. Phys. 100, 073506 (2006).

55. H. Hirori, T. Tachizaki, O. Matsuda, and O. B. Wright, "Electron dynamics in chromium probed with 20 fs optical pulses," Phys. Rev. B 68, 113102 (2003)

56. Y. S. Touloukian, R. W. Powell, C. Y. Ho, and P. G. Klemens, eds., Thermal Conductivity-Metallic Elements and Alloys, Vol. 1 of Thermophysical Properties of Matter (IFI/Plenum, 1970).

57. Y. S. Touloukian and E. H. Buyco, eds., Specific Heat-Metallic Elements and Alloys, Vol. 4 of Thermophysical Properties of Matter (IFI/Plenum, 1970).

58. Z. Lin, L. V. Zhigilei, and V. Celli, "Electron-phonon coupling and electron heat capacity of metals under conditions of strong electron phonon nonequilibrium," Phys. Rev. B 77, 075133 (2008).

59. B. A. Auld, Acoustic Fields and Waves in Solids, 2nd ed (Krieger, 1990) 
60. As discussed in relation to Fig. 6 , the permittivity at the probe light wavelength can be retrieve $\bar{d}$ from the interferometric data. This fitted value for the probe light lies close to that of corresponding ellipsometry data, so we also adopted the ellipsometry data value for the pump light.

61. J. G. Fujimoto, J. M. Liu, and E. P. Ippen, "Femtosecond laser interaction with metallic tungsten and nonequilibrium electron and lattice temperatures," Phys. Rev. Lett. 53, 1837-1840 (1984).

62. S. D. Brorson, A. Kazeroonian, J. S. Moodera, D. W. Face, T. K. Cheng, E. P. Ippen, M. S. Dresselhaus, and G. Dresselhaus, "Femtosecond room-temperature measurement of the electron-phonon coupling constant $\lambda$ in metallic superconductors," Phys. Rev. Lett. 64, 2172-2175 (1990).

63. We used fitted values for the permittivity of the $\mathrm{W}$ film at the probe wavelength rather than from the literature, $5.3+16.2 i$ (see [44]), or from ellipsometry measurement, $3.1+4.1 i$. These differences in permittivity may be attributable to impurities in the $\mathrm{W}$ film.

64. The spectrum in strain, obtained by multiplication of that of the displacement by $i \omega$, shows significant noise above $\sim 30 \mathrm{GHz}$.

65. C. K. Jones and J. A. Rayne, "Ultrasonic attenuation in tungsten and molybdenum up to $1 \mathrm{Gc} / \mathrm{s}$," Phys. Lett. 13, 282-283 (1964).

66. M. J. G. Lee, J. M. Perz, and J. Plotnick, "Electronic attenuation of longitudinal acoustic phonons in tungsten," Phys. Rev. Lett. 48, 30-33 (1982).

67. G. D. Mansfeld, S. G. Alekseev, and I. M. Kotelyansky, "Acoustic HBAR spectroscopy of metal (W, Ti, Mo, Al) thin films," in Ultrasonics Symposium, Vol. 1 (IEEE, 2001) pp. 415-418. 